\title{
Novel prognostic genes of diffuse large B-cell lymphoma revealed by survival analysis of gene expression data
}

\author{
This article was published in the following Dove Press journal: \\ OncoTargets and Therapy \\ 18 November 2015 \\ Number of times this article has been viewed
}

Chenglong $\mathrm{Li}^{1,2}$

Biao Zhu', ${ }^{1,2}$

Jiao Chen ${ }^{1,2}$

Xiaobing Huang ${ }^{1,2}$

'Department of Hematology, Sichuan Academy of Medical Sciences \&

Sichuan Provincial People's Hospital, Chengdu, Sichuan, People's Republic of China; ${ }^{2}$ Department of Hematology, Affiliated Medical School of University of Electronic Science and Technology, Chengdu, Sichuan, People's Republic of China
Correspondence: Xiaobing Huang Department of Hematology, Sichuan Academy of Medical Sciences \& Sichuan Provincial People's Hospital, 32 West Second Section First Ring

Road, Chengdu, Sichuan 6I0072,

People's Republic of China

Tel +8602887394243

Email huangxbds@।63.com
Objective: This study aimed to identify prognostic genes for diffuse large B-cell lymphoma (DLBCL), using bioinformatic methods.

Methods: Five gene expression data sets were downloaded from the Gene Expression Omnibus database. Significance analysis of microarrays algorithm was used to identify differentially expressed genes (DEGs) from two data sets. Functional enrichment analysis was performed for the DEGs with the Database for Annotation, Visualization and Integration Discovery (DAVID). Survival analysis was performed with the Kaplan-Meier method using function survfit from package survival of $R$ for the other three data sets. Cox univariate regression analysis was used to further screen out prognostic genes.

Results: Thirty-one common DEGs were identified in the two data sets, mainly enriched in the regulation of lymphocyte activation, immune response, and interleukin-mediated signaling pathway. Combined with 47 DLBCL-related genes acquired by literature retrieval, a total of 78 potential prognostic genes were obtained. Cases from the other three data sets were used in hierarchical clustering, and the 78 genes could cluster them into several subtypes with significant differences in survival curves. Cox univariate regression analysis revealed 45, 33, and eleven prognostic genes in the three data sets, respectively. Five common prognostic genes were revealed, including $L C P 2, T N F R S F 9$, FUT8, IRF4, and TLE1, among which $L C P 2$, FUT8, and TLE1 were novel prognostic genes.

Conclusion: Five prognostic genes of DLBCL were identified in this study. They could not only be used for molecular subtyping of DLBCL but also be potential targets for treatment.

Keywords: diffuse large B-cell lymphoma, gene expression profile, differentially expressed genes, survival analysis, subtype

\section{Introduction}

Diffuse large B-cell lymphoma (DLBCL) is one of the most common types of non-Hodgkin lymphoma, which occurs primarily in older individuals. It is an aggressive tumor. R-CHOP, an improved form of cyclophosphamide, doxorubicin, vincristine, and prednisone (CHOP) with the addition of rituximab, is a standard treatment for DLBCL.

Many subtypes of the lymphoid neoplasms are established based on the World Health Organization classification system, and DLBCL is the most common type in Asians. ${ }^{1}$ However, classification merely based on morphology and clinical information is difficult and thus a considerable percentage of cases are not classified. Gene expression profiling studies have attempted to distinguish heterogeneous groups of DLBCL from each other. ${ }^{2-4}$ For instance, by gene expression profile, two groupings of germinal center B-cell-like and the activated B-cell-like were identified as two DLBCL subtypes in the current World Health Organization classification. ${ }^{5}$ The study by Lenz et $\mathrm{al}^{6}$ provides genetic evidence that the DLBCL subtypes are distinct diseases that use different 
oncogenic pathways. Obviously, DNA microarrays provide a better understanding of the biology of DLBCL and advance the development of novel diagnostic tools. ${ }^{7}$

Meanwhile, many genes with prognostic effect have been reported in DLBCL, such as BCL2 ${ }^{8}$ and BCL6. ${ }^{9} \mathrm{Hu}$ et $\mathrm{al}^{10}$ suggested that MYC/BCL2 coexpression, rather than cell-of-origin classification, is a better predictor of prognosis in patients with DLBCL treated with R-CHOP. Additionally, Gratzinger et $a^{11}$ reported the prognostic value of vascular endothelial growth factor and vascular endothelial growth factor receptors in DLBCL patients treated with anthracycline-based chemotherapy. Besides, Hussain et al ${ }^{12}$ found that $\mathrm{X}$-linked inhibitor of apoptosis expression is a poor prognostic factor for DLBCL.

Due to the heterogeneity of DLBCL, more works are necessary to advance molecular subtyping as well as to discover the prognostic genes. In this study, two gene expression data sets were analyzed to identify differentially expressed genes (DEGs), which were regarded as potential prognostic genes for DLBCL, and to ascertain whether these genes would be used to well distinguish the subtypes of DLBCL in other three expression profile data sets.

\section{Methods}

\section{Gene expression data}

All the five gene expression data sets were downloaded from the Gene Expression Omnibus.

1) The data set of GSE32918 $8^{13,14}$ collected gene expression profiles of 172 DLBCL samples. The platform of Illumina GPL8432 (Illumina HumanRef-8 WG-DASL v3.0) was used. It included a total of 294 sequencing data since some samples were sequenced repeatedly.

2) The data set of GSE10846 $6^{15,16}$ included gene expression profiles of 181 clinical samples from chemotherapytreated patients and 233 clinical samples from rituximabchemotherapy-treated patients. The platform was Affymetrix GPL570 (Affymetrix Human Genome U133 Plus 2.0 Array). A total of 416 gene expression data were included.

3) The data set of GSE1 $1318^{6}$ consisted of gene expression profiles of 203 DLBCL samples, based on the platform of Affymetrix GPL570.

4) The data set of GSE932 $7^{17}$ collected gene expression profiles of 36 DLBCL samples and eight reactive lymph nodes samples, which were used as controls. The platform of GPL6011 (CNIO Human Oncochip 1.0, 1.2, and 2.0) was used.

5) The data set of GSE30881 ${ }^{18}$ contained gene expression profiles of 23 DLBCL samples and ten healthy controls, in order to investigate the changes in NF- $\kappa \mathrm{B}$ pathway activation. The platform was Affymetrix GPL3738 (Affymetrix Canine Genome 2.0 Array).

\section{Pretreatment of raw data}

Probes were mapped to genes according to the annotation files. For a gene corresponding to more than one probe, the average probe value was calculated as the gene expression value for the specific gene. ${ }^{19}$ Subsequently, $\log 2$ conversion and quantile normalization ${ }^{20}$ were applied on the data.

A total of 4,356 and 16,454 unique genes were identified in GSE9327 and GSE30881, respectively. Both GSE10846 and GSE11318 were obtained using GPL570, and a total of 20,693 unique genes were acquired. Besides, 18,403 unique genes were identified in GSE32918.

\section{Clinical information}

The expression profiles of GSE10846 and GSE11318 provided clinical information such as age, sex, stage, lactate dehydrogenase (LDH) level, extranodal versus nodal presentation, treatment, subtype, survival time, and survival status. GSE32918 described age, sex, treatment, subtype, survival time, and survival status. According to these three data sets, we found that "stage" could well separate samples into different groups with diverse survival time while "age", "sex", and "treatment" could not.

\section{Screening of DEGs}

Significance analysis of microarrays algorithm ${ }^{21}$ was adopted to screen out DEGs. It can reduce the false-positive rate in multiple testing via controlling false discovery rate. Relative difference (statistic $d$ ) is calculated as follows:

$$
d=\frac{X_{1}^{\prime} \mid X_{2}^{\prime}}{S+s_{0}}
$$

Statistic $d$ measures the relative differences in gene expression levels, and it is the corrected $t . X_{1}^{\prime}$ represents the average expression level of a gene under certain state, $X_{2}^{\prime}$ represents the average expression level of a gene under another state, and $s$ represents the variance of a gene.

Adjusted $P$-value $<0.05$ and $\log \mid$ fold change $\mid>1.5$ were set as the threshold to select the DEGs.

\section{Functional enrichment analysis}

Gene ontology enrichment analysis and Kyoto Encyclopedia of Genes and Genomes pathway enrichment analysis were performed for the DEGs with DAVID ${ }^{22}$ to examine the potential altered functions and pathways of these DEGs. False discovery rate $<0.05$ was set as the cutoff. 


\section{Survival analysis}

Kaplan-Meier method (K-M method; product-limit method) is suitable for analysis with small sample size. The analysis procedure is as follows: 1) Put the samples in ascending order according to the survival time, rank $i=1,2, \ldots, n$. 2) List the number of surviving at the beginning of each time point (in fact, a short time). 3) Calculate the probability of death at each time point $q$ and survival probability $p(p=1-q)$. 4) Calculate the survival rate $S(t i)$ for each time point, which equals to the product of each survival probability from the starting point to $t i . S(t i)=p_{1} \times p_{2} \times p_{3} \ldots p_{t i}$. Finally, plot survival curves with survival time in abscissa and survival rate in ordinate.

Survival analysis was performed with function survfit from package survival of $R \cdot{ }^{23}$ Difference in survival curves for two groups was analyzed with log-rank method using function survdiff from package survival. ${ }^{24}$

\section{Screening of risk factors}

Cox univariate regression analysis was carried out using function coxph from package survival to screen out risk factors related to survival. ${ }^{25}$ The formula is as follows:

$$
h(t, x)=h_{0}(t) \exp \left(\beta_{i} \times x i\right)
$$

$h_{0}(t)$ is the basic risk function, the risk function when all covariates $X_{1}, X_{2}, \ldots, X_{\mathrm{m}}$ are 0 or under standard conditions, and it is generally unknown. $h(t, x)$ represents the risk function when each covariate $X$ is given a fixed value, and it is proportional to $h_{0}(t)$. Therefore, the model is also known as the proportional hazard model. $X_{1}, X_{2}, \ldots, X_{\mathrm{m}}$ are covariates while $\beta_{1}, \beta_{2}, \ldots, \beta_{\mathrm{m}}$ are regression coefficients. When the regression coefficient $\beta_{i}>0$, that is, the risk ratio $>1$, it indicates that the covariate is a risk factor. The greater the covariate is, the shorter the survival time is. When the regression coefficient $\beta_{i}<0$, that is, the risk ratio $<1$, it indicates that the covariate is a protective factor, so the greater the covariate is, the longer the survival time is.

\section{Results \\ Differentially expressed genes and enriched biological functions}

According to the aforementioned criteria, a total of 437 DEGs were identified in DLBCL from the data set GSE9327 and 1,457 DEGs from the data set GSE30881. Thirty-one overlapping genes were selected out and functional enrichment analysis was performed for these genes, which are mainly involved in the regulation of lymphocyte activation, immune response, and interleukin-mediated signaling pathway (Figure 1), suggesting that the 31 DEGs were closely associated with the development of DLBCL.

Moreover, 47 DLBCL-related genes were acquired via literature retrieval. ${ }^{2,15,26-31}$

\section{Survival analysis result}

The 31 DEGs and 47 DLBCL-related genes were combined and a total of 78 potential prognostic genes were obtained, which were used to classify samples with diverse survival time from other three data sets.

1) In the data set of GSE10846, 71 out of the 78 genes were detected. Using hierarchical clustering, the 71 genes could well cluster the 416 DLBCL samples into four subtypes (Figure 2A). The differences in survival curves of the

\section{GO functional enrichment of 31 DEGs}

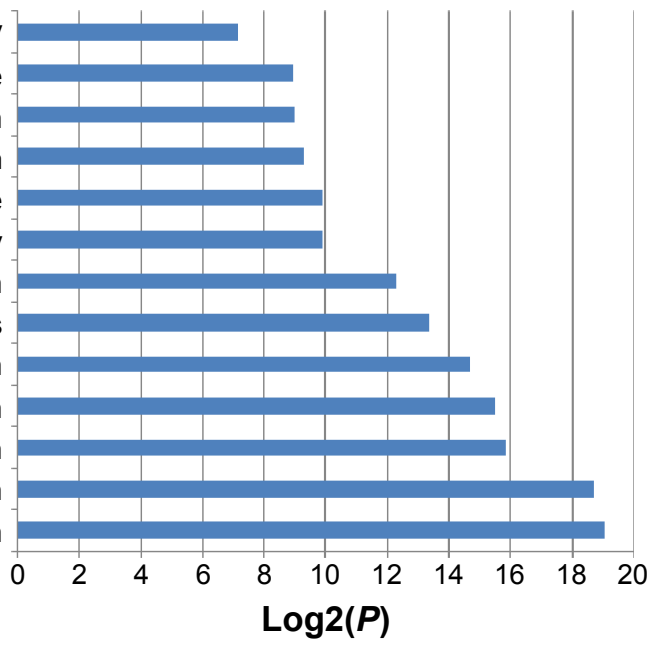

Figure I Functional enrichment analysis result for the 3 I differentially expressed genes (DEGs) (top 20 gene ontology [GO] terms ranked by the significance). Notes: $X$-axis represents the adjusted $P$-value transformed by log2, and $Y$-axis denotes the enriched $G O$ terms.

Abbreviation: IL, interleukin. 

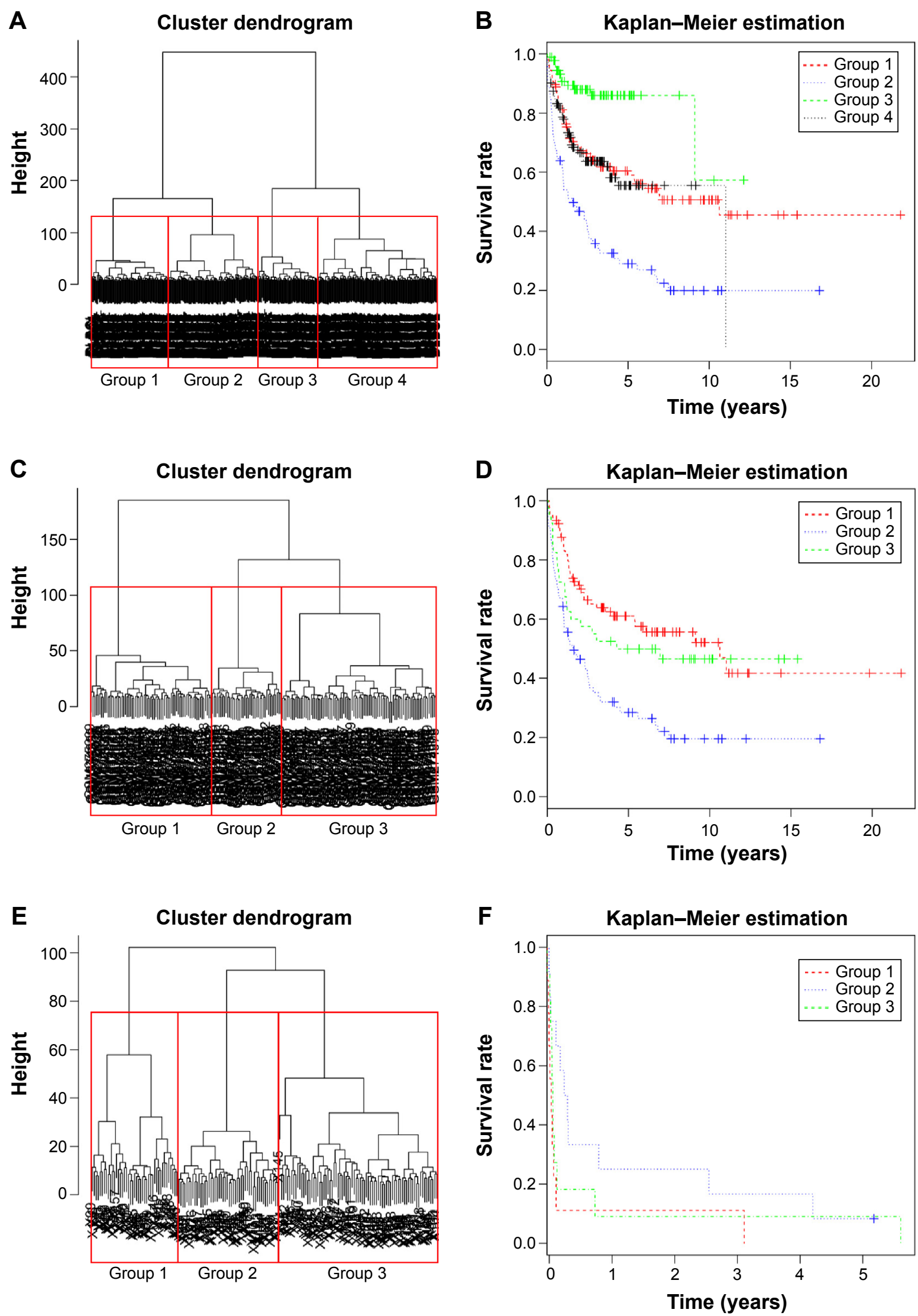

Figure 2 Subtyping of diffuse large B-cell lymphoma (DLBCL) in three gene data sets using the 78 predicted and curated DLBCL-related genes.

Notes: (A, C, and E) Hierarchical clustering that denotes the subtypes of DLBCL clustered by the 78 genes in the gene data sets of GSEI0846, GSEII3I8, and GSE329I8, respectively; (B, D, and F) Kaplan-Meier survival curves of the subtypes in the gene data sets of GSEI0846, GSEII3I8, and GSE329I8, respectively. 


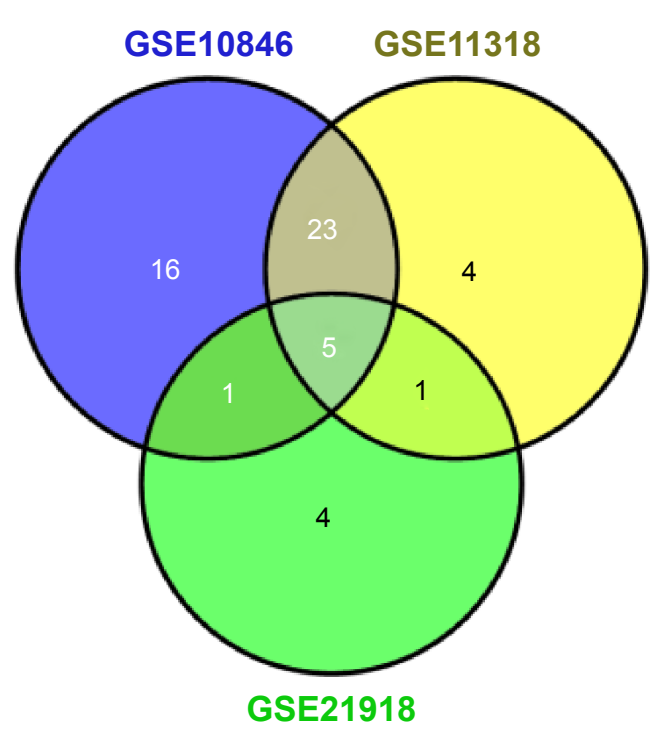

Figure 3 Venn diagram of the prognostic genes from three gene expression data sets (GSEI0846, GSEI I3।8, and GSE21918).

four subtypes were found to be significant $\left(P=7.65 \mathrm{e}^{-11}\right.$; Figure 2B).

2) In the data set of GSE11318, 71 out of the 78 genes were detected. Using hierarchical clustering, the 71 genes could well classify the 203 DLBCL samples into three subtypes (Figure 2C). The difference in survival curves of the three subtypes was found to be significant $(P=7.5 \mathrm{e}-05$; Figure 2D).

3) In the data set of GSE32918,69 out of the 78 genes were detected. Some samples were sequenced repeatedly, and thus average expression levels were calculated as the final values. Using hierarchical clustering, the 69 genes could cluster the 172 DLBCL samples into three subtypes (Figure 2E). The difference in survival curves of the three subtypes was found to be significant $(P=0.013$; Figure 2F).

\section{Prognostic genes}

The correlation between each gene and the survival of DLBCL patients was calculated with Cox univariate regression analysis to further screen out genes with prognostic value. In the data set of GSE10846, 45 genes were found to have significant prognostic effect, while in GSE11318, 33 genes had prognostic effect, and in GSE32918, eleven genes showed prognostic value. Five prognostic genes were common among the three data sets (Figure 3; Table 1). According to the coefficient, lymphocyte cytosolic protein 2 (LCP2) and tumor necrosis factor receptor superfamily member 9 (TNFRSF9) might be related to poor prognosis while fucosyltransferase 8 (FUT8), interferon regulatory factor 4 (IRF4), and transducin-like enhancer of split 1 (TLE1) might bring in favorable prognosis.

\section{Discussion}

In this study, five gene expression data sets were downloaded from the Gene Expression Omnibus. Thirty-one common DEGs were identified from two gene expression data sets, mainly enriching in the regulation of lymphocyte activation, immune response, and interleukin-mediated signaling pathway, which were closely associated with the development of DLBCL. Combined with 47 DLBCL-related genes acquired by literature retrieval, 78 potential prognostic genes were obtained, which could successfully cluster the DLBCL samples from another three gene expression data sets into several subtypes with significant differences in survival. Prognostic genes were screened out via Cox univariate regression analysis, and five common genes were acquired, such as LCP2, TNFRSF9, FUT8, IRF4, and TLE1.

TNFRSF $^{32}$ and IRF4 ${ }^{33}$ are two known prognostic genes of DLBCL. TNFRSF9 is a member of the TNF-receptor superfamily that can induce proliferation in peripheral monocytes. Alizadeh et $\mathrm{al}^{32}$ indicate that expression levels of LIM domain only 2 (LMO2) and TNFRSF9 powerfully predict the overall survival in patients with DLBCL. TNFRSF9 can also serve as the target to treat DLBCL. The study by Houot et $\mathrm{al}^{34}$ demonstrates that anti-CD137 therapy has a potent antilymphoma activity in a mouse model. IRF4 belongs to the interferon regulatory factor (IRF) family of transcription factors. Salaverria et $\mathrm{al}^{35}$ report that translocations activating IRF4 identify a subtype of germinal center-derived B-cell lymphoma affecting predominantly children and young adults. Therefore, it may be a therapeutic target of DLBCL. ${ }^{36}$

Table I Five common prognostic genes

\begin{tabular}{|c|c|c|c|c|c|c|}
\hline \multirow[t]{2}{*}{ Gene names } & \multicolumn{2}{|c|}{ GSEI 0846} & \multicolumn{2}{|c|}{ GSE I I 3 | 8} & \multicolumn{2}{|c|}{ GSE329 I 8} \\
\hline & $P$-value & Coefficient & $P$-value & Coefficient & $P$-value & Coefficient \\
\hline FUT8 & I.07E-05 & 0.340027 & 0.010986 & 0.247543 & 0.035247 & 0.276876 \\
\hline IRF4 & 0.000575 & 0.249417 & 0.00936 & 0.261503 & 0.039336 & 0.235775 \\
\hline$L C P 2$ & 0.027791 & -0.18334 & 0.026033 & -0.24189 & 0.009313 & -0.50978 \\
\hline TLEI & 0.00152 & 0.222874 & $3.28 \mathrm{E}-05$ & 0.354751 & 0.001212 & 0.357821 \\
\hline TNFRSF9 & $3.65 \mathrm{E}-08$ & -0.38752 & 0.005578 & $-0.2464 I$ & 0.045842 & -0.23852 \\
\hline
\end{tabular}


LCP2, FUT8, and TLE1 may be novel prognostic genes of DLBCL. LCP2 plays a positive role in promoting T-cell development and activation as well as mast cell and platelet function. FUT8 is an enzyme belonging to the family of fucosyltransferases. It may contribute to the malignancy of cancer cells and to their invasive and metastatic capabilities. ${ }^{37}$ Chen et $\mathrm{al}^{38}$ found that FUT8 is upregulated during epithelialmesenchymal transition via the transactivation of $\beta$-catenin/ lymphoid enhancer-binding factor (LEF)-1. Based on these instances, we speculated that FUT8 might exert a similar role in DLBCL and thus contributes to the metastasis of DLBCL. TLE1 is a multitasked transcriptional corepressor that acts through the acute myelogenous leukemia 1, Wnt, and Notch signaling pathways. Promoter CpG island hypermethylation-associated inactivation of TLE1 has been observed in DLBCL. ${ }^{39}$ Fraga et $\mathrm{al}^{40}$ further point out that TLE1 epigenetic inactivation contributes to the development of hematologic malignancies by disrupting critical differentiation and growth-suppressing pathways. However, the exact role of TLE1 in DLBCL remains to be explored. We supposed that more researches may unveil clinical applications of the three genes.

Overall, five critical genes with prognostic effect were disclosed in DLBCL via bioinformatic analysis of existing gene expression data. Two out of the five genes have been reported while the other three are novel predictors. Further researches on these genes can benefit molecular subtyping and also provide potential therapeutic targets of DLBCL.

\section{Highlights}

1) A set of 31 common DEGs were identified from two gene expression data sets.

2) Totally, 78 potential prognostic genes were suggested be used for subtyping of DLBCL.

3) Five prognostic genes, including three novel ones, were identified in DLBCL.

\section{Disclosure}

The authors report no conflicts of interest in this work.

\section{References}

1. Morton LM, Wang SS, Devesa SS, Hartge P, Weisenburger DD, Linet MS. Lymphoma incidence patterns by WHO subtype in the United States, 1992-2001. Blood. 2006;107(1):265-276.

2. Alizadeh AA, Eisen MB, Davis RE, et al. Distinct types of diffuse large B-cell lymphoma identified by gene expression profiling. Nature. 2000;403(6769):503-511.

3. Hoefnagel JJ, Dijkman R, Basso K, et al. Distinct types of primary cutaneous large B-cell lymphoma identified by gene expression profiling. Blood. 2005;105(9):3671-3678.
4. Visco C, Li Y, Xu-Monette ZY, et al. Comprehensive gene expression profiling and immunohistochemical studies support application of immunophenotypic algorithm for molecular subtype classification in diffuse large B-cell lymphoma: a report from the International DLBCL Rituximab-CHOP Consortium Program Study. Leukemia. 2012;26(9): 2103-2113

5. Xu Q, Tan C, Ni S, et al. Identification and validation of a two-gene expression index for subtype classification and prognosis in diffuse large B-cell lymphoma. Sci Rep. 2015;5:10006.

6. Lenz G, Wright GW, Emre NC, et al. Molecular subtypes of diffuse large B-cell lymphoma arise by distinct genetic pathways. Proc Natl Acad Sci U S A. 2008;105(36):13520-13525.

7. Lossos IS, Morgensztern D. Prognostic biomarkers in diffuse large B-cell lymphoma. J Clin Oncol. 2006;24(6):995-1007.

8. Dunleavy K, Wilson WH. Differential role of BCL2 in molecular subtypes of diffuse large B-cell lymphoma. Clin Cancer Res. 2011; 17(24):7505-7507.

9. Winter JN, Weller EA, Horning SJ, et al. Prognostic significance of Bcl-6 protein expression in DLBCL treated with CHOP or R-CHOP: a prospective correlative study. Blood. 2006;107(11):4207-4213.

10. Hu S, Xu-Monette ZY, Tzankov A, et al. MYC/BCL2 protein coexpression contributes to the inferior survival of activated B-cell subtype of diffuse large B-cell lymphoma and demonstrates high-risk gene expression signatures: a report from The International DLBCL Rituximab-CHOP Consortium Program. Blood. 2013;121(20):4021-4031.

11. Gratzinger D, Zhao S, Tibshirani RJ, et al. Prognostic significance of VEGF, VEGF receptors, and microvessel density in diffuse large B cell lymphoma treated with anthracycline-based chemotherapy. Lab Invest. 2008;88(1):38-47.

12. Hussain AR, Uddin S, Ahmed M, et al. Prognostic significance of XIAP expression in DLBCL and effect of its inhibition on AKT signalling. J Pathol. 2010;222(2):180-190.

13. Barrans SL, Crouch S, Care MA, et al. Whole genome expression profiling based on paraffin embedded tissue can be used to classify diffuse large B-cell lymphoma and predict clinical outcome. Br J Haematol. 2012; 159(4):441-453.

14. Care MA, Cocco M, Laye JP, et al. SPIB and BATF provide alternate determinants of IRF4 occupancy in diffuse large B-cell lymphoma linked to disease heterogeneity. Nucleic Acids Res. 2014;42(12):7591-7610.

15. Lenz G, Wright G, Dave SS, et al; Lymphoma/Leukemia Molecular Profiling Project. Stromal gene signatures in large-B-cell lymphomas. N Engl J Med. 2008;359(22):2313-2323.

16. Cardesa-Salzmann TM, Colomo L, Gutierrez G, et al. High microvessel density determines a poor outcome in patients with diffuse large B-cell lymphoma treated with rituximab plus chemotherapy. Haematologica. 2011;96(7):996-1001.

17. Ruiz-Vela A, Aggarwal M, de la Cueva P, et al. Lentiviral (HIV)-based RNA interference screen in human B-cell receptor regulatory networks reveals MCL1-induced oncogenic pathways. Blood. 2008;111(3): 1665-1676.

18. Mudaliar MA, Haggart RD, Miele G, et al. Comparative gene expression profiling identifies common molecular signatures of NF-kappaB activation in canine and human diffuse large B cell lymphoma (DLBCL). PLoS One. 2013;8(9):e72591.

19. Ma H, Schadt EE, Kaplan LM, Zhao H. COSINE: condition-specific sub-network identification using a global optimization method. Bioinformatics. 2011;27(9):1290-1298.

20. Ferrari F, Bortoluzzi S, Coppe A, et al. Novel definition files for human GeneChips based on GeneAnnot. BMC Bioinformatics. 2007;8:446.

21. Larsson $\mathrm{O}$, Wahlestedt $\mathrm{C}$, Timmons JA. Considerations when using the significance analysis of microarrays (SAM) algorithm. BMC Bioinformatics. 2005;6:129.

22. Dennis G Jr, Sherman BT, Hosack DA, et al. DAVID: database for annotation, visualization, and integrated discovery. Genome Biol. 2003;4(5):3.

23. Xu Y, Gao X, Wang Z. [Nonparametric method of estimating survival functions containing right-censored and interval-censored data]. Sheng Wu Yi Xue Gong Cheng Xue Za Zhi. 2014;31(2):267-272. 
24. Jones MP, Crowley J. A general class of nonparametric tests for survival analysis. Biometrics. 1989;45(1):157-170.

25. Andersen PAG. R, Cox's regression model for counting processes, a large sample study. Ann Stat. 1982;10:20.

26. Rosenwald A, Wright G, Chan WC, et al; Lymphoma/Leukemia Molecular Profiling Project. The use of molecular profiling to predict survival after chemotherapy for diffuse large-B-cell lymphoma. $N$ Engl J Med. 2002;346(25):1937-1947.

27. Shipp MA, Ross KN, Tamayo P, et al. Diffuse large B-cell lymphoma outcome prediction by gene-expression profiling and supervised machine learning. Nat Med. 2002;8(1):68-74.

28. Lossos IS, Czerwinski DK, Alizadeh AA, et al. Prediction of survival in diffuse large-B-cell lymphoma based on the expression of six genes. N Engl J Med. 2004;350(18):1828-1837.

29. Cai YD, Huang T, Feng KY, Hu L, Xie L. A unified 35-gene signature for both subtype classification and survival prediction in diffuse large B-cell lymphomas. PLoS One. 2010;5(9):e12726.

30. Rimsza LM, Unger JM, Tome ME, Leblanc ML. A strategy for full interrogation of prognostic gene expression patterns: exploring the biology of diffuse large B cell lymphoma. PLoS One. 2011;6(8):e22267.

31. Wright G, Tan B, Rosenwald A, Hurt EH, Wiestner A, Staudt LM. A gene expression-based method to diagnose clinically distinct subgroups of diffuse large B cell lymphoma. Proc Natl Acad Sci U S A. 2003; 100(17):9991-9996.

32. Alizadeh AA, Gentles AJ, Alencar AJ, et al. Prediction of survival in diffuse large B-cell lymphoma based on the expression of 2 genes reflecting tumor and microenvironment. Blood. 2011;118(5):1350-1358.

33. Richards KL, Motsinger-Reif AA, Chen HW, et al. Gene profiling of canine B-cell lymphoma reveals germinal center and postgerminal center subtypes with different survival times, modeling human DLBCL. Cancer Res. 2013;73(16):5029-5039.
34. Houot R, Goldstein MJ, Kohrt HE, et al. Therapeutic effect of CD137 immunomodulation in lymphoma and its enhancement by Treg depletion. Blood. 2009;114(16):3431-3438.

35. Salaverria I, Philipp C, Oschlies I, et al; Molecular Mechanisms in Malignant Lymphomas Network Project of the Deutsche Krebshilfe, German High-Grade Lymphoma Study Group, Berlin-FrankfurtMünster-NHL trial group. Translocations activating IRF4 identify a subtype of germinal center-derived B-cell lymphoma affecting predominantly children and young adults. Blood. 2011;118(1):139-147.

36. Shaffer AL, Emre NC, Romesser PB, Staudt LM. IRF4: immunity. malignancy! therapy? Clin Cancer Res. 2009;15(9):2954-2961.

37. Ito Y, Miyauchi A, Yoshida H, et al. Expression of alpha1,6fucosyltransferase (FUT8) in papillary carcinoma of the thyroid: its linkage to biological aggressiveness and anaplastic transformation. Cancer Lett. 2003;200(2):167-172.

38. Chen CY, Jan YH, Juan YH, et al. Fucosyltransferase 8 as a functional regulator of nonsmall cell lung cancer. Proc Natl Acad Sci USA. 2013; 110(2):630-635.

39. Castellano G, Torrisi E, Ligresti G, et al. Yin Yang 1 overexpression in diffuse large B-cell lymphoma is associated with B-cell transformation and tumor progression. Cell Cycle. 2010;9(3):557-563.

40. Fraga MF, Berdasco M, Ballestar E, et al. Epigenetic inactivation of the Groucho homologue gene TLE1 in hematologic malignancies. Cancer Res. 2008;68(11):4116-4122.
OncoTargets and Therapy

\section{Publish your work in this journal}

OncoTargets and Therapy is an international, peer-reviewed, open access journal focusing on the pathological basis of all cancers, potential targets for therapy and treatment protocols employed to improve the management of cancer patients. The journal also focuses on the impact of management programs and new therapeutic agents and protocols on

\section{Dovepress}

patient perspectives such as quality of life, adherence and satisfaction. The manuscript management system is completely online and includes a very quick and fair peer-review system, which is all easy to use. Visit http://www.dovepress.com/testimonials.php to read real quotes from published authors. 